\title{
Properties of CPW in the Sub-mm Wave Range and Its Potential to Radiate
}

\author{
J. Zehentner, J. Macháč \\ Czech Technical University, Technická 2, 16627 Prague 6, Czech republic \\ Tel +420 2 24352273, Fax +420 2 24355865, E-mail zeh@mbox.cesnet.cz
}

\section{Introduction}

In recent years, new technologies of monolithic integrated circuits have been pushing utilization of the $\mathrm{CPW}$ as far as the sub-mm wave range [1-3]. Additional new findings on CPW characteristics and behaviour have in this way been revealed step by step. Our paper contributes to this process.

Detailed investigation of possible higher order leaky waves that may occur on the CPW has allowed us to make a new interpretation of the measured dispersion characteristics [2] of the $\mathrm{CPW}$, made on a high resistivity Si substrate, in the frequency range up to $700 \mathrm{GHz}$. For a microwave designer, it is very important to know the frequency up to which the line transmits only the bound wave. This upper cutoff frequency has been determined, and data are available for a wide range of line cross-sectional dimensions and substrate permittivities. CPW also has the potential to radiate space leaky waves. We report theoretical identification of the space leaky waves on the CPW. Tzuang and Lin discovered, and later experimentally verified, this type of leaky wave two years ago on the suspended microstrip line $[4,5]$.

\section{Dispersion characteristics of CPW and comparison with experiments}

The coplanar waveguide, the cross-section of which is shown in the inset of Fig. 1, can leak power at high frequencies. Tsuji, Shigesawa and Oliner investigated leaky waves on this open unboxed line in [6]. In [7] we identified higher order leaky waves on the slotline, which represent the simultaneous power leakage into the corresponding number of surface waves. Two adjacent leaky waves may exist in a quite wide overlapping frequency band. As for the CPW, we have shown that on this line higher order surface leaky waves can occur successively. However, the frequency band of simultaneous propagation of two adjacent waves is now relatively narrow. These conclusions result from spectral domain analysis (SDA) of the CPW, assuming infinite conductivity and zero thickness of the conductors. Ground strips are laterally infinitely wide.

Pfeifer et al [2] measured at frequencies ranging up to $670 \mathrm{GHz}$ the propagation constant of the CPW, with finite but sufficiently wide ground strips, fabricated on a silicon substrate. The measured data are plotted in Fig. 1 and 2, and compared with our calculated values. Heinrich's model [8] provides a good description of the effective permittivity, which is equal to the square of the normalized phase constant, at frequencies lower than $80 \mathrm{GHz}$, as follows from [2]. At higher frequencies, Frankel's semiempirical analytic dispersion formula [1] fits well with the measured record [2]. Interpretation of the measured dispersion characteristic based on our leaky wave concept is as follows. Bound wave propagation stops and 1st leaky wave excitation starts in the vicinity of $81 \mathrm{GHz}$. This wave is replaced by the 2nd leaky wave in the vicinity of $129 \mathrm{GHz}$, etc. The 8th leaky wave is substituted for the 7th wave around 700 GHz. This means that the field propagating over the CPW in the sub-mm wave range consists of consecutive higher order surface leaky waves, complemented probably by residual waves [9], depending on the source. 
Besides attenuation due to leakage and the lossy substrate, we also have to account for conductor losses. For a $0.4 \mu \mathrm{m}$ thick electroplated Au conductor with conductivity of $3.10^{7}$ $\mathrm{S} / \mathrm{m}$, the CPW attenuation constant was calculated according to [8], assuming a lossless substrate. In leakage constant computation (SDA), the imaginary part of the silicon frequency dependent permittivity [10] was used. After adding to the leakage constant, including substrate lossess, the attenuation constant raised due to metallization losses, the total attenuation constant compares well with the measured data. Apparently the main component of the losses is the leakage of power, followed by the influence of metallization, and finally the dielectric losses are negligible.

\section{Upper cutoff frequency of bound wave propagation on the CPW}

The bound wave and the 1st leaky wave can propagate simultaneously on the CPW in a relatively narrow band. The frequency at which the 1 st leaky wave sets in determines $f_{C}$, the upper cutoff frequency of pure bound wave propagation. We have computed $f_{C}$ for a set of $\mathrm{w} / \mathrm{h}, \mathrm{s} / \mathrm{h}, \varepsilon_{\mathrm{r}}$ combinations when $\mathrm{w} / \mathrm{h}, \mathrm{s} / \mathrm{h} \in(0.1,1.9)$ and $\varepsilon_{\mathrm{r}}=2.25,3.75,5,7,9.8,11,15$. The normalized upper cutoff frequency $h / \lambda_{C}=f_{C} h / c$ is plotted in Fig. 3 for selected permittivities. When, e. g., $\varepsilon_{\mathrm{r}}=15$, a function

$\left(\mathrm{h} / \lambda_{\mathrm{C}}\right)=\mathrm{A}_{1}+\left[\mathrm{A}_{2}+\mathrm{A}_{3}(\mathrm{w} / \mathrm{h})^{-\mathrm{A}_{4}}\right]^{-\mathrm{A}_{5}}-\mathrm{A}_{6} \mathrm{e}^{-\mathrm{A}_{7}\left|(\mathrm{w} / \mathrm{h})-\mathrm{A}_{8}\right|^{\mathrm{A}_{9}}}$

where $A_{1}, A_{2}, \ldots A_{9}$ are simple functions of $s / h$, fits such set of curves with an error of less than $0.65 \%$ at all one hundred calculated points. Knowledge of this frequency cut down facilitates CPW design.

\section{Space leakage from CPW}

We have investigated the properties of the CPW by the method of moments in the spectral domain. Analysis in the source-free region provides particular components of the total field propagating over the line. First, we recalculated the dispersion characteristics of a slotline with infinite wide conductors, in order to assure ourselves that the integration being conducted to the space leaky wave is right. It turned out that our values differ negligibly from those published in Fig. 3 of [11]. We therefore applied the same procedure in the CPW analysis.

The dispersion characteristics of the independent space leaky waves on the CPW are shown in Fig. 4. All curves were computed with the same number of basis functions. A number of branches were found subsequently along the frequency axis, but only five of them are plotted in Fig. 4. These characteristics correspond to the simultaneous space and surface leakage, since the branch cut and the pole corresponding to the $\mathrm{TM}_{0}$ surface wave were taken into account in their calculation. We believe that waves corresponding to the characteristics quoted here are an analogy of the space-leaky modes carrying dominant-mode-like currents on suspended microstrip revealed by Tzuang and Lin in [4]. The electric field within the slots of the CPW for those space leaky waves greatly resembles that of the dominant bound wave.

\section{Conclusions}

We have shown that the main part of the field propagating over the CPW in the sub-mm wave range consists of consecutively excited higher order surface leaky waves. In addition, residual waves and/or space leaky waves may accompany them, depending on the source. If 
we exclude space leaky waves, the frequency band of pure bound wave propagation is determined by the frequency at which the 1 st surface leaky wave sets in. We have provided this frequency for a wide range of line dimensions and substrate permittivities. We have found a new solution of the dispersion equation for the CPW that is relevant for a space leaky wave with a field distribution within the slots, as is the case for the dominant bound mode. As we know, waves of this type have already been discovered in [4] on a suspended microstrip line. We consider that our findings contribute to the general discussion on leakage effects on open transmission lines.

\section{References}

[1] M. Y. Frankel, S. Gupta, J. A. Valmanis, G. A. Mourou: Terahertz Attenuation and Dispersion Characteristics of Coplanar Transmission Lines. IEEE Trans. Microwave Theory Tech., vol. MTT-39, pp 910-916, June 1991.

[2] T. Pfeifer, H. M. Heiliger, T. Loffer, H. G. Roskos, H. Kurz: Picosecond Optoelectronic On-Wafer Characterisation of Coplanar Waveguides on High-Resistivity $\mathrm{Si}$ and $\mathrm{Si} / \mathrm{SiO}_{2}$ Substrates. Microelectronic Engineering, vol. 31, pp. 385-395, 1996.

[3] F. Steinhagen, W. H. Haydl, T. Krems, W. Marsetz, R. Locher, C. Wild, P. Kidl, A. Hulsmann, T. v. Kerssenbrok, P. Heide: Microwave Properties of Coplanar Transmission Lines and Filters on Diamond from 1-120 GHz. 1998 IEEE MTT-S Int. Microwave Sym. Digest, vol. 2, pp. 1065-1068, Baltimore, MD, 1998.

[4] C.-K. C. Tzuang, C.-C. Lin: Space-wave leaky mode carrying dominant-mode-like current distributions. IEEE MTT-S Int. Microwave Sym. Digest, pp. 643-646, Baltimore, MD, 1998.

[5] C.-K. C. Tzuang: Leaky Mode Perspective of Printed Antenna. 1998 Asia-Pacific Microwave Conference Proceedings, vol. 3, pp. 1471-1478, Yokohama.

[6] N. Tsuji, H. Shigesawa, A. A. Oliner: New interesting leakage behaviour on coplanar waveguides of finite and infinite widths. IEEE Trans. Microwave Theory Tech., vol. MTT-39, pp. 2130-2136, Dec. 1991.

[7] J. Zehentner, J. Macháć, M. Migliozzi: Upper cutoff frequency of the bound wave and new leaky wave on the slotline. IEEE Trans. Microwave Theory Tech., vol. MTT-46, pp. 378386, Apr. 1998.

[8] W. Heinrich: Quasi-TEM Description of MMIC Coplanar Lines Including ConductorLoss Effects. IEEE Trans. Microwave Theory Tech., vol. MTT-41, pp. 45-52, Jan. 1993.

[9] M. J. Freire, F. Mesa, D. R. Jackson: The Residual-Wave Current Excited on a PrintedCircuit Line: A Source of Spurious Effects. 29th European Microwave Conference Proceedings, vol. 3, pp. 114-117, Munich 1999.

[10] M. N. Afsar, K. J. Button: Precise Millimeter-Wave Measurements of Complex Refractive Index, Complex Dielectric Permittivity and Loss Tangent of GaAs, $\mathrm{Si}, \mathrm{SiO}_{2}$, $\mathrm{Al}_{2} \mathrm{O}_{3}, \mathrm{BeO}$, Macor, and Glass. IEEE Trans. Microwave Theory Tech., vol. MTT-31, pp. 217223, Feb. 1983.

[11] J.-W. Sheen, Y.-D. Lin: Propagation Characteristics of the Slotline First Higher Order Mode. IEEE Trans. Microwave Theory Tech., vol. MTT-46, pp. 1774-1781, Nov. 1998 


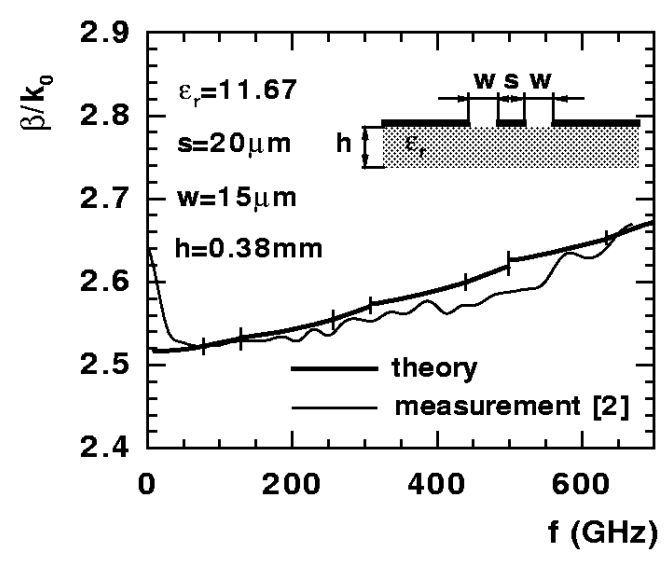

Fig. 1 The calculated and measured normalized phase constant of the CPW as a function of frequency

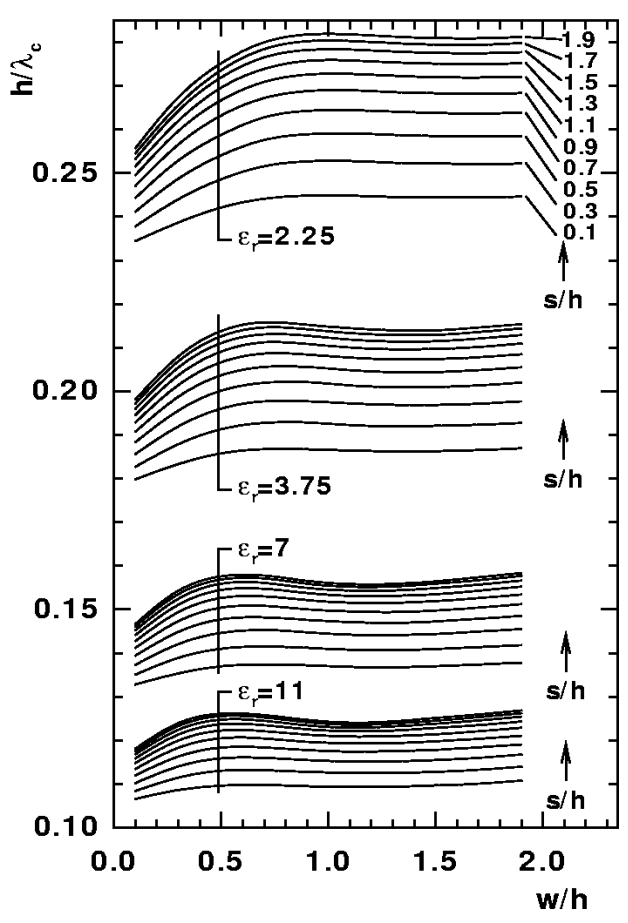

Fig. 3 The normalized upper cutoff frequency $h / \lambda_{C}$ of the CPW

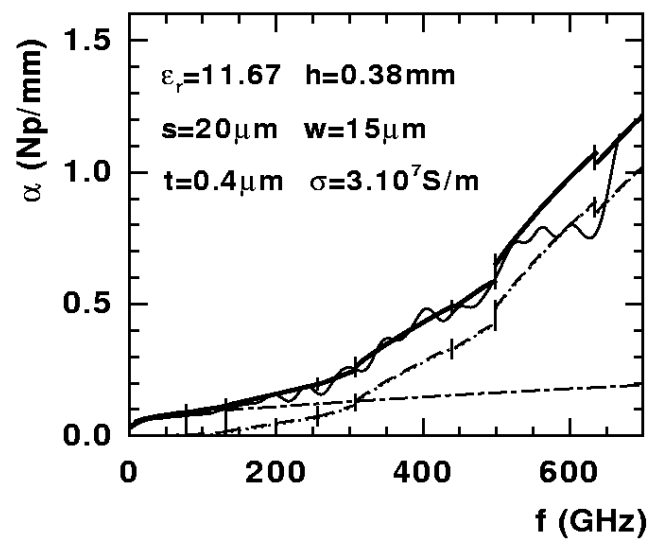

Fig. 2 The attenuation/leakage constant of the CPW: measured [2] — calculated:

only leakage from the lossless line $\ldots . . \cdots \cdots$, leakage and losssy substrate - - - - , only lossy metallization [8] $-\cdot-\cdot-\cdot$, total losses

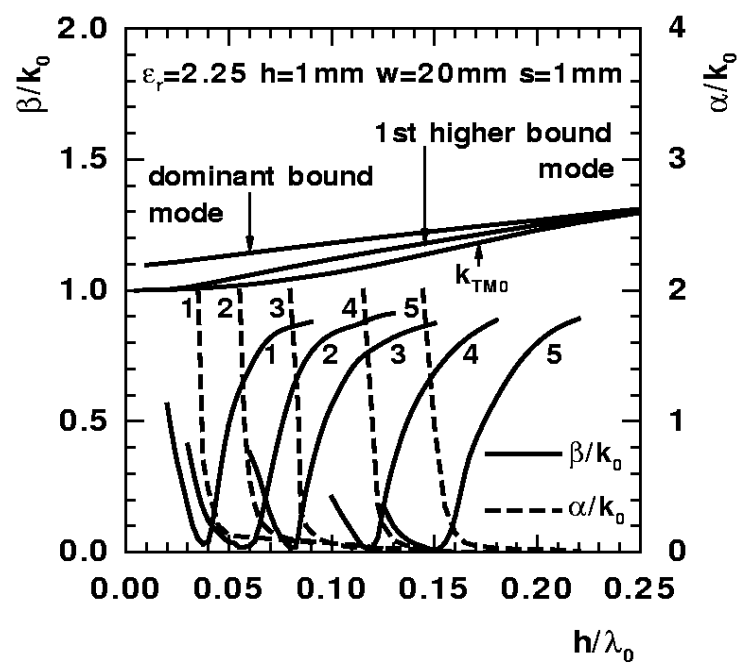

Fig. 4 Dispersion characteristics of the CPW 